\title{
Filigrane
}

Écoutes psychothérapiques

\section{Les rêves en héritage. La possibilité et l'impossibilité de rêver}

\section{René Kaës}

Volume 16, numéro 1, printemps 2007

Les hauts lieux et non-lieux du rêve I

URI : https://id.erudit.org/iderudit/016173ar

DOI : https://doi.org/10.7202/016173ar

Aller au sommaire du numéro

Éditeur(s)

Revue Santé mentale au Québec

ISSN

1192-1412 (imprimé)

1911-4656 (numérique)

Découvrir la revue

Citer cet article

Kaës, R. (2007). Les rêves en héritage. La possibilité et l'impossibilité de rêver. Filigrane, 16(1), 5-14. https://doi.org/10.7202/016173ar

\section{Résumé de l'article}

Dans La polyphonie du rêve (2002), l'auteur a exploré certains aspects de l'expérience onirique lorsque le rapport des rêveurs à leurs rêves est traversé par les rêves d'autres rêveurs. Dans cet article, la recherche se poursuit et porte sur le désir d'attribuer ses rêves à autrui et sur l'angoisse d'être dépossédé de son propre espace onirique. La clinique, relayée par l'analyse d'une nouvelle de J. Irving, montre que ces angoisses sont le plus souvent liées à la difficulté de constituer un espace interne qui soit pensable comme étant propre au sujet. L'empiètement ou l'intrusion de cet espace onirique crée un collage d'enveloppes psychiques, dont il n'est possible de se détacher qu'en se déchirant de la peau et la psyché maternelles. 


\title{
Les rêves en héritage. La possibilité et I'impossibilité de rêver
}

\section{rené kaës}

\begin{abstract}
Dans La polyphonie du rêve (2002), l'auteur a exploré certains aspects de l'expérience onirique lorsque le rapport des rêveurs à leurs rêves est traversé par les rêves d'autres rêveurs. Dans cet article, la recherche se poursuit et porte sur le désir d'attribuer ses rêves à autrui et sur l'angoisse d'être dépossédé de son propre espace onirique. La clinique, relayée par l'analyse d'une nouvelle de J. Irving, montre que ces angoisses sont le plus souvent liées à la difficulté de constituer un espace interne qui soit pensable comme étant propre au sujet. L'empiètement ou l'intrusion de cet espace onirique crée un collage d'enveloppes psychiques, dont il n'est possible de se détacher qu'en se déchirant de la peau et la psyché maternelles.
\end{abstract}

$\mathrm{D}$ ans L'interprétation des rêves, Freud a dégagé certains principes du fonctionnement du rêve, de ses processus et du travail psychique qu'il requiert pour s'accomplir. Il a proposé une conception forte de sa fonction intrapsychique: le rêve est la réalisation hallucinatoire d'un désir inconscient, d'un désir de la veille et d'un désir sexuel infantile refoulé. Freud a donné au rêve un statut épistémologique et méthodologique éminent: le rêve est la voie royale d'accès à l'Inconscient, et c'est dans cette position qu'il est objet d'interprétation, dans le cadre de la cure, c'est-à-dire dans le transfert. Il n'est pas inutile de souligner que Freud découvre les processus de la formation du rêve dans les limites internes de l'espace psychique ; c'est dans cet espace qu'il en dégage les fonctions et qu'il apporte une réponse à la question de son sens.

Ce que Freud a pensé du rêve ne décrit pas toutes les expériences oniriques dont peut rendre compte la psychanalyse. Aujourd'hui le rêve n'est plus envisagé seulement comme réalisation hallucinatoire du désir et comme voie royale d'accès à l'Inconscient. Si nous continuons à comprendre le rêve à l'intérieur de l'espace de la réalité intrapsychique où il est nécessairement produit par un rêveur singulier, l'étude de ses conditions internes, de ses processus, de ses contenus et de son sens montre que le rêve est aussi une expérience créatrice, réparatrice, transformatrice.

En outre, l'intérêt pour l'espace intrapsychique du rêve s'est déplacé vers son émergence et sa fonction dans l'espace transféro-contretransférentiel. Les psychanalystes ont découvert une autre façon de concevoir le rêve sous l'effet conjoint des transformations de la pratique de la cure et des théories qui en rendent compte ou qui les inspirent.

Pour ce qui me concerne, la clinique de la cure individuelle m'a conduit à porter attention à la formation, à la consistance et au destin des rêves croisés de l'analyste 
et de l'analysant, aux interférences entre leurs rêves de séances et au statut des rêves contre-transférentiels de l'analyste. De tels rêves s'observent également dans les thérapies psychanalytiques de la famille, du couple et des groupes. Ce sont ces dispositifs de travail, dérivés de celui de la cure et aménagés en fonction de leurs objets, qui ont permis de formuler de nouvelles vues sur les limites de l'espace intrapsychique, en l'ouvrant davantage sur ses bords et sur ses interférences avec les espaces psychiques d'autres sujets.

Dans mes recherches sur le rêve, et notamment dans La polyphonie du rêve (2002), j'ai voulu explorer comment le rêve se forme à la fois dans l'espace de la réalité intrapsychique, où il est nécessairement produit par un rêveur singulier, et dans un autre espace, que l'on peut nommer interpsychique, où il trouve une autre source de fomentation et où il développe des effets spécifiques. $\mathrm{Si}$, dans ces deux espaces, le rêve est une création individuelle, «égoïste» (Freud) c'est-à-dire au service du Moi, il ne se nourrit pas aux mêmes sources, ne s'organise pas de la même manière ni pour les mêmes fonctions dans chacun d'entre eux.

En portant l'analyse sur les rêves qui surgissent dans le cadre de la cure individuelle et dans les dispositifs psychanalytiques centrés sur les configurations de liens, j'ai essayé de répondre à la question suivante: comment penser l'expérience onirique lorsque le rapport des rêveurs à leurs rêves est traversé par les rêves d'autres rêveurs?

Toutefois, la clinique m'a indiqué une autre perspective sur cette question, et je n'en ai pas fait mention dans La polyphonie du rêve. Il s'agit du désir d'attribuer ses rêves à autrui et de l'angoisse d'être dépossédé de son propre espace onirique. J'ai commencé à explorer quelques aspects de cette relation du rêveur à son propre rêve et aux rêves des autres. Les données cliniques que j'ai recueillies ne semblent pas invalider, au contraire, les trois principales hypothèses que $\mathrm{j}$ 'ai prises comme fil conducteur pour conduire les recherches exposées dans La polyphonie du rêve, et que je vais résumer.

\section{Un espace onirique commun et partagé entre plusieurs rêveurs}

J'ai supposé un espace onirique commun et partagé, où le rêve est travaillé par et dans une multiplicité d'espaces, de temps, de sens et de voix. J'en ai donné plusieurs exemples. Un de mes patients a longtemps rêvé - et désiré rêver les «mêmes» rêves que son frère. Ce que l'analyse de ses rêves d'enfance a mis en évidence, dans le transfert, c'est d'abord le désir de rêver les mêmes rêves dans un espace onirique commun et partagé avec son frère. Le désir de se rencontrer dans le même espace onirique a cherché alors, comme aujourd'hui dans la cure, sa voie de réalisation dans les objets oniriques communs. L'espace onirique commun redoublait l'espace nocturne du même lit partagé, il met au jour les enjeux incestuels du lien qui unit mon patient à son frère: leur stratégie mobilise les inductions dans le travail de la veille pour faire surgir dans chacun de leur propre rêve le désir de l'un de s'y retrouver avec l'autre. Leurs rêves ne sont pas identiques, ils ont des traits communs et partagés, mais chacun rêve son propre rêve soutenu par le même désir: rejoindre le frère dans l'espace onirique imaginaire commun. 
Ces rêves s'élaborent dans l'étoffe onirique du lien. Chacun est relié avec les autres dans la matière onirique commune et partagée dont le lien est tissé. Le rêve se constitue à partir de la formation d'un espace onirique pluriel, commun et partagé. L'analyse des rêves en groupe met au jour cet espace dans lequel se représentent les formations de l'inconscient des sujets dans leur rencontre avec l'autre (plus d'un autre).

En mettant l'accent sur l'espace onirique partagé et commun à plusieurs rêveurs, je ne perds pas de vue que le rêve est la création d'un rêveur, qu'il est «égoïste». Je dis que le premier, l'espace interne du rêve, communique avec le second: l'espace onirique partagé et commun à plusieurs rêveurs. Mais je suppose que les conditions de la formation du rêve et son organisation, par résonance identificatoire et fantasmatique avec les rêves d'autres rêveurs, s'inscrivent dans un autre espace psychique, qui englobe le premier et interfère avec lui, un espace où le rêve «repose sur l'inconnu».

\section{Les deux ombilics du rêve}

La seconde hypothèse avec laquelle j'ai travaillé est que le rêve a deux berceaux, deux ombilics. J'ai repris la métaphore freudienne de l'ombilic du rêve ancré dans le mycélium psychosomatique ${ }^{1}$ pour introduire l'idée qu'il existe un second ombilic du rêve, un lieu de passage où il plonge dans l'inconscient des liens interpsychiques les plus anciens. Ces deux ombilics reposent sur «l'inconnu» d'où les rêves surgissent. Ce qui, dans le désir du rêve, surgit du plus profond de l'inconscient est ancré dans l'expérience corporelle et interpsychique, il transite à travers l'ombilic du rêve.

C'est de cet ombilic interpsychique, qui forme dans la matrice onirique maternelle des deux frères, que surgissent les matériaux de leurs rêves et c'est sur ce mycélium onirique qu'ils s'alimentent. Pour autant chacun des rêveurs est en contact avec cette autre part de l'inconscient chevillée dans l'expérience corporelle. La capacité de rêver, le désir du rêve, le travail du rêve, les fonctions et le sens du rêve dans l'expérience onirique sont déterminés, selon des proportions variables, dans chacun de ces espaces.

\section{La polyphonie du rêve}

J'ai proposé une troisième hypothèse : le rêve est une polyphonie de plusieurs «écritures », il est travaillé par et dans une multiplicité d'images, d'espaces, de temps, de sens et de voix. Dans chaque rêve, plusieurs voix sont à l'œuvre, restes diurnes et nocturnes de ses propres rêves et de ceux de quelques autres. La matière première du rêve est dans cette matière polyphonique. Le travail du rêve les transforme, à moitié effacés et à moitié lisibles, comme dans un palimpseste, amalgamés dans un texte qui ne devient déchiffrable que si nous disposons d'une hypothèse suffisamment précise pour les décrypter et pour restituer l'expérience onirique dans ses procédés de création poétique ${ }^{2}$.

L'hypothèse de la polyphonie du rêve nous conduit dans une «fabrique de rêves » où plusieurs espaces oniriques s'interpénètrent, où plusieurs rêveurs se font 
signe et se font entendre de plusieurs rêveurs, de plusieurs auditeurs, internes et externes. La polyphonie du rêve décrit comment, à partir des deux ombilics du rêve et de la formation d'un espace onirique pluriel, commun et partagé, le rêve s'organise comme une combinaison de plusieurs voix ou de plusieurs parties de voix, de plusieurs images, de plusieurs tableaux repliés et qui forment un ensemble.

Dans La polyphonie du rêve, j'ai souvent soutenu que le désir de rêver dans l'espace onirique commun est probablement une réalisation du désir le plus ancien de l'être humain, et j'ai parlé de la satisfaction de rêver ensemble, d'inclure l'autre dans son propre espace onirique et d'être inclus dans le rêve de l'autre. Venons-en maintenant à des données cliniques qui semblent contredire ces hypothèses, ou du moins les interroger selon une autre perspective.

\section{Le désir d'attribuer ses rêves à autrui et l'angoisse d'être dépossédé de son propre espace onirique}

Dès qu'elle s'était allongée sur le divan, Madame C. avait exprimé sa crainte que les rêves de ceux qui étaient déjà en analyse avec moi ne l'imprègnent : «je ne voudrais pas faire leur rêves », me dit-elle. Plus tard, revenant sur sa phobie, elle ajoutera qu'elle avait été, au début de son adolescence, fort impressionnée par les histoires de succubes et d'incubes. «Rêver les rêves des autres, c'est comme avoir un enfant incestueux, sans s'en rendre compte, comme dans ces histoires». Sa crainte n'était pas sans rapport avec ce qui avait été éveillé dans son désir par ces histoires.

Lors de cette première séance, je me suis demandé si j'avais bien fait de lui proposer le divan: à ce moment-là, je n'entrevoyais dans ce qu'elle me disait qu'un moment de dépersonnalisation, comme il arrive quelquefois lors de la première séance sur le divan avec des personnalités mal assurées dans leurs limites internes. Par la suite, ces premières paroles se chargèrent d'autres sens. Ce qu'elle m'avait dit lors des entretiens préliminaires me revint en mémoire: sa mère lui imposait de lui livrer sa vie intime, et notamment de lui parler de ses rêves. C'est une des raisons qui me conduisirent à ne lui proposer le divan qu'après une assez longue période de séances en face-à-face. Elle m'avait beaucoup parlé de l'intrusion maternelle.

Elle n'avait jamais pu raconter ses rêves à personne et, durant longues périodes, elle ne rêvait pas ou alors elle pensait qu'elle ne se souvenait plus de ses rêves. Après la séance, elle occupait une bonne dizaine de secondes à relisser le divan, à rétablir le volume du coussin, à en effacer le creux que sa tête y avait fait, elle ne voulait pas laisser de traces.

Ce rituel me fit penser à un de mes patients, lui aussi doté d'une mère intrusive. Pendant plusieurs années, il était venu à ses séances nimbé d'une enveloppe olfactive très forte, faite d'une odeur de camphre et de benjoin. Il lui fallait laisser des traces. Il lui importait de se faire reconnaître malade de ce lien tyrannique qui le liait à sa mère: plus il demandait de soins, plus sa mère exerçait son emprise sur 
lui, plus il s'en rendait dépendant. Lui aussi disait ne plus rêver, mais sa peau se couvrait régulièrement d'eczéma ; c'était alors la trace qui marquait une visite que sa mère lui faisait, ou d'une visite qu'il lui rendait. Lorsque nous parvînmes à établir ce lien, puis les relations entre son absence de rêve et les atteintes contre sa peau, il fit un rêve: sa femme lui donnait un bain trop chaud et le langeait dans un drap trop froid. Mais il pensa que c'était là non pas son propre rêve, mais un rêve de sa mère, et qu'elle lui avait choisi sa femme à sa propre image, de telle sorte qu'elle continue à exercer sur lui son emprise.

Dans les groupes, j' avais eu aussi affaire à ce fantasme «ne pas occuper la place d'un autre », de crainte que les pensées secrètes de celle ou de celui qui avait précédé le participant à cette place n'influencent ses propres pensées.

Dans tous ces cas, qu'il s'agisse de la crainte ou du désir que les autres influencent ses propres rêves ou ses propres pensées, un fantasme de transmission impose cette représentation. J'ai désigné par fantasme de transmission (1993) un scénario inconscient dans lequel le sujet convoque les protagonistes de son histoire et de sa préhistoire, et où il se représente comme innocent d'un héritage qui lui aurait été transmis comme un contenu psychique à la fois étranger et familier, par un contemporain ou par un ancêtre.

Cette représentation fantasmatique de processus et d'objets transmis directement par un autre est toujours marquée par la prévalence de l'identification projective. L'angoisse de la dépossession est régulièrement corrélée avec une relation intrusive de la mère avec son enfant et avec une culpabilité intense de celui-ci à l'égard de sa mère, en raison de la haine que suscite cette intrusion mais aussi du besoin de maintenir cette relation.

Que les rêves s'inscrivent dans un espace psychique commun et partagé est une question dont j'ai tenté de montrer les conditions et les effets. Mais autre chose est le fantasme que les rêves que nous faisons sont les rêves des autres, et non les siens propres. C'est cette méconnaissance qui fait question. En attribuant le rêve à un autre, le sujet s'innocente de son propre désir. On peut alors faire l'hypothèse que la culpabilité, sous diverses formes, paranoïde et dépressive, est au cœur de cette représentation par laquelle le sujet se dégage de son propre espace psychique subjectif. On peut aussi supposer que l'identification projective est ici à l'œuvre pour tenter de contrôler l'espace onirique de l'autre. Dans l'exemple du groupe que je viens d'évoquer, le fantasme de transmission couvre défensivement un fantasme de meurtre du rival (de ses pensées) qui occupait précédemment la place que va choisir le sujet.

Alors que je travaillais sur ces questions, j'ai lu une nouvelle de John Irving, fort bien intitulée «Les rêves des autres ». Cette nouvelle n'apporte évidemment pas une réponse exhaustive aux questions que pose le fantasme et le paradoxe des rêves que nous faisons et qui seraient ceux des autres, mais elle ouvre quelques pistes de réflexion. Je la résume. 


\section{Une nouvelle de John Irving : «Les rêves des autres».}

Le héros, Fred, ne se souvenait pas d'avoir rêvé avant que sa femme ne le quitte. Elle l'avait quitté parce qu'il ne cessait de tomber amoureux d'autres femmes, le plus souvent assez bécasses. C'est seulement après leur séparation que Fred avait ressenti la blessure des dix années de vie conjugale sans rêves, alors que sa femme rêvait «comme une forcenée». Elle ne lui racontait jamais ses rêves, mais le matin, il cherchait sur son visage la trace de ses secrets nocturnes. Lorsqu'il se retrouva seul, avec le chien, il ne put dormir dans le lit qu'il avait partagé avec sa femme. Il avait choisi le canapé du séjour, il y dormait mal, sans rêves, jusqu'à cette nuit où il se figura qu'il était en train de vomir dans une voiture. Sa passagère, Mrs Beal, le frappait et lui criait de les ramener à la maison. Fred comprit qu'il rêvait et il tint pour assuré qu'il avait fait le rêve de Mr Beal qui, précisément avait souvent tourné de l'œil sur leur canapé. Le rêve de Fred avait été fait par Mr Beal, qui «l'avait laissé en héritage pour le prochain dormeur au sommeil agité».

Il passe alors la nuit dans le lit de son fils. Dédoublé et réuni en "père et fils», comme dans un personnage composite, il rêve qu'il est dans un champ à la merci d'un gros serpent, mi-dragon, mi-serpent de mer. La bête s'élance contre la poitrine du rêveur qui, paralysé par l'angoisse, ne peut pas crier. «À l'autre bout du champ, écrit J. Irving, Fred se voyait comme son fils le voyait». Le père, debout au-dessus d'un feu qui couvait, pissait sur les cendres, une vapeur d'urine s'élevait autour de lui et il n'entendait pas crier son fils.

Les rêves de son fils étant trop explicites et triviaux, Fred décide non sans précaution de revenir dormir dans le lit conjugal. Il y fait un des rêves de sa femme: il se regardait dans un miroir en pied, mais c'était elle qu'il voyait, elle était nue. Il vint à l'esprit de Fred qu'il rêvait pour lui-même, dans un sursaut de son désir pour elle. Mais la femme qu'il voyait dans le miroir était vieille et laide, elle pleurait et plaquait contre elle des masses de vêtements qui tombaient ensuite à ses pieds et sur lesquels elle finit par s'effondrer. Il retrouve le souvenir d'un voyage avec sa femme: une vieille femme était tombée dans un escalier, et il l'avait retrouvée au pied de celui-ci, sa robe noire en bouchon sur ses cuisses. Dans le miroir de son rêve, sa femme s'était endormie sur sa pile de vêtements. Il se souvint que c'est dans cette position qu'il l'avait retrouvée la nuit de sa première infidélité.

Fred continue à penser qu'il ne peut dormir sans hériter des rêves d'autrui. Il voudrait faire les siens, et aussi se délivrer de la fatigue et de l'excitation que lui impose sa faculté miracle. Quel lieu trouver dans sa propre maison pour cela: le vieux canapé sur lequel le labrador avait élu domicile, mais alors faire un rêve de chien? Sans doute le canapé avait aussi accueilli quelques baby-sitters: mais le risque était grand de faire les rêves du chien au lieu des songes suaves des jeunes filles.

Lorsque son père mourut, il rendit visite à sa mère. À sa surprise, elle prit le canapé pour elle et lui offrit le lit parental: «avec son potentiel de rêves homériques, le lit le terrifiait..., il allait le prendre aux rets de rêves bien 
embarrassants ». Il rêva un rêve de sa mère, et il en fut soulagé en vertu d'une règle qu'il s'était donnée: on ne rêve jamais les rêves des morts. Il fut surpris de la verdeur du rêve maternel: son père, en érection, gambadait sous la douche, se préparant à des ébats agiles, inventifs et acrobatiques. Il rêvait l'affection « chaude et humide de sa mère pour l'homme en rut qu'il n'avait jamais soupçonné chez son père ». C'était son premier rêve dans la peau d'une femme, il venait de rêver comment sa mère jouissait.

Fred se dit qu'il ne rêvait pas encore ses propres rêves, mais des rêves d'occasion, il ne savait pas que l'on peut rêver ce qui n'est jamais arrivé.

\section{Commentaires}

Irving sait bien que son héros est le rêveur de ses propres rêves. Dans chacun d'entre eux, il montre le dédoublement du rêveur: Fred-Beal, le père-fils, Fred-sa femme, Fred le voyeur de la scène primitive qui lui donne à connaître, dans la peau d'une femme, la jouissance maternelle et l'art érotique de son père. Mais il attribue ses rêves à un(e) autre, parce que chacun de ses doubles le met en contact avec la partie de lui-même qu'il refuse ou qu'il doit méconnaître. Dans son premier rêve, ce qu'il vomit est son propre espace interne, c'est pourquoi il attribue le rêve à cet autre minable qu'il est aux yeux de sa femme et que représente l'évanescent $\mathrm{Mr}$ Beal, préservant à ce prix son intégrité narcissique, sa puissance phallique et la connaissance de la haine de sa femme pour ses amours nombreuses et légères. $\mathrm{Ce}$ n'est qu'en s'éveillant du rêve qu'il lui attribue que Fred saura, note Irving, «combien ses infidélités l'avaient fait se prendre en haine elle-même». Le rêve a cette fois fonctionné selon l'identification projective normale, il lui donne accès à la connaissance intime, encore partielle et voilée, du monde interne de l'autre. Le rêve qui précède, celui où il est à la fois le père et le fils montre combien il est préoccupé de maintenir sa puissance phallique urétrale, identifié en cela à son fils, mais incapable d'en entendre les cris d'effroi. Enfin, le rêve attribué à la mère est bien la réalisation de son fantasme d'identification féminine dans cette scène de séduction où triomphe le père, est celui qui lui donne accès à la pensée de sa propre sexualité, immature, terne et maladroite.

Remarquable est la composition de la nouvelle: d'abord deux rêves attribués à des hommes (Mr Beal — son double minable — et son fils, ce sont des rêves de persécution sur fond d'angoisse de castration. Viennent ensuite les deux rêves attribués à des femmes (sa femme, sa mère) avec lesquels il récupère ses identifications bisexuelles et la culpabilité dépressive.

Se penser l'héritier direct des rêves d'autrui permet de ne pas être confronté au processus de subjectivation de son propre espace psychique. À vrai dire la question est plus complexe, si l'on considère ce que nous apprend la clinique des rêves dans les groupes et les familles. Lorsque les rêves circulent dans le même espace psychique (onirique) commun et partagé, au point que, comme le dit si justement J. Saramago dans son Essai sur l'aveuglement, c'est le rêve lui-même qui rêve et non chaque rêveur, nous pouvons être assurés que nous avons affaire à des espaces 
psychiques intrusés, réciproquement envahis, mais dans lesquels la capacité de rêver est encore suffisamment préservée pour qu'elle constitue le dernier recours contre l'arasement complet de la subjectivité. Assurément, la nouvelle d'Irving ne contient pas d'éléments qui évoqueraient autre chose que cet envahissement de l'espace onirique de Fred par les rêves «des autres », selon la théorie du rêveur.

\section{Retour à la clinique}

Dans le cas des deux patients dont j'ai parlé au début de cette étude, l'emprise intrusive de la mère était l'objet d'une angoisse et d'un désir déplacé sur mes patients et sur moi. Chez le patient, l'emprise se retourne dans l'empreinte ou l'imprégnation olfactive (laisser des traces imprégnantes) sur moi et sur les patients suivants. Chez la patiente, l'emprise ou l'empiètement maternel se retourne dans l'emprise qu'elle exerce sur le creux laissé par le corps sur le divan. Pour l'un comme pour l'autre, ne pas rêver, c'est se soustraire à l'intrusion et à l'emprise maternelle, mais d'une manière si radicale que cela revient à s'amputer de son propre espace onirique: à la place de cet enveloppe défaillante, une peau qui suinte, se dessèche et s'écaille, qui ne peut contenir et filtrer les perceptions ${ }^{3}$. Ou une enveloppe imperméable, intacte, sans trace ni mémoire, et une phobie des contacts.

Mon idée première, selon laquelle avec de tels patients, on pourrait avoir affaire à un moment de dépersonnalisation en début de cure ou à des noyaux de délire d'influence, n'était pas sans intérêt pour l'écoute et la conduite de la cure: il fallait entendre que les «autres» auxquels étaient attribués ces rêves insoutenables, inacceptables, étaient des représentants du Moi du sujet, rejetés, expulsés dans des autres. Ces autres figuraient leur propre aliénation dans la place qui leur avait été assignée par leur mère, de telle sorte qu'ils ne pouvaient constituer un espace interne dans lequel il pouvait se passer quelque chose qu'ils puissent penser comme leur étant propre. Cet espace avait été empiété, intrusé, collé à celui de leur mère d'une manière telle qu'ils ne pouvaient s'en détacher qu'en déchirant leur peau et leur psyché d'avec la peau et la psyché de leur mère. Chacun de mes patients avait eu de graves problèmes de séparation, de sevrage, d'acceptation des pertes fécales.

Pouvoir reconnaitre que l'on peut avoir ses propres rêves suppose bien plus qu'une relative clôture de l'espace psychique: cela suppose que la capacité de rêver ait été désirée, soutenue et nourrie par la mère ou par la personne qui en accomplit la fonction. C'est ce qui manque à la représentation des patients dont j'ai parlé au début de cette étude. Comme sans doute à Fred, qui commence son aventure après la séparation d'avec sa femme.

À la fin de son parcours dans la nouvelle d'Irving, Fred continuera sans doute à penser que certains lieux gardent l'empreinte des rêves des autres. Il les recherchera ou les évitera. Il continuera à assimiler l'autre au lieu qu'il occupe, et il mettra son autre interne hors de lui, sans le reconnaitre. Irving nous dit que son héros n'envisageait pas «que les rêves qu'il faisait pouvaient lui appartenir — qu'il 
n'oserait jamais s'approcher davantage de lui-même». Il venait seulement « de se découvrir une faille de vulnérabilité incurable». Mais il aura été saisi, un bref instant, par une pensée qui pourrait le guérir: «Il lui arrivait de penser que, si seulement il avait réussi à faire ses rêves à lui, il n'aurait pas eu besoin de passer à l'acte et de se lancer dans ces lamentables liaisons presque tous les ans ». Rêver limite la mise en acte, c' est ce que soutiennent notamment M. Khan, J.-B. Pontalis, P.-C. Racamier.

Lorsque l'espace interne et l'espace de rêve ne sont pas suffisamment établis, les rêves ne peuvent pas se rêver à l'intérieur: le rêve au-dehors est son issue. Une autre issue, paradoxale, est que le rêveur ne parvient à rêver qu'en se faisant, dans le fantasme de transmission, l'héritier passif des rêves des autres.

Nous voici donc reconduit à notre question principale, qui s'enrichit de cette clinique de la dépossession et de la séparation. Dans tous les cas, nous avons affaire à un espace interpsychique, où le rêve se fabrique, se rêve, et se raconte dans le récit qui en est fait à un autre. Dans cet espace, la capacité onirique d'un autre - la mère - et de plus d'un autre - le groupe, la famille, le couple — est un facteur essentiel de la formation du développement des fonctions du rêve.

$\mathrm{Au}$ cours des conversations que j'ai eues avec C. Neri à propos de ces recherches, nous nous sommes posé à plusieurs reprises la question de savoir si la création et la mise en œuvre de l'espace onirique précèdent ou suivent la capacité de rêver.

Cette question n'est pas seulement théorétique, elle a aussi d'importantes implications cliniques. En effet, si la construction de l'espace onirique précède la possibilité de rêver, le psychanalyste, dans la cure comme dans le groupe, doit privilégier son déploiement plutôt que le travail d'interprétation des rêves. Il doit surtout beaucoup écouter et éviter les interventions intempestives qui peuvent interférer négativement sur la mise en œuvre de cet espace. Je pense que la création de l'espace onirique commun et partagé précède la capacité de rêver du sujet. Je pense, suivant Bion en cela, que le jeu entre les espaces oniriques de plusieurs rêveurs est une étape décisive dans la formation de la capacité de rêver. La clinique que j'ai élaborée dans La polyphonie du rêve, comme dans les analyses que je viens d'exposer, me montrent que la restauration de la capacité de rêver est un autre effet de l'expérience onirique commune et partagée sur le rêveur considéré isolément. Elle est repérable dans la clinique, à la condition que ne se produise ni intrusion ni empiètement.

rené kaës

32, cours de la liberté

F. 69003 lyon kaes.rene@free.fr 
Filigrane, printemps 2007

\section{Notes}

1. «Dans les rêves les mieux interprétés, écrit Freud (1900, G. W. II-III, p. 530), on doit souvent laisser un endroit dans l'ombre parce que l'on remarque, à l'interprétation, qu'il y a là un enchevêtrement (ein Knäuel) de pensées du rêve qui ne saurait se défaire, mais qui n'apporte aucune contribution supplémentaire au contenu du rêve. C'est l'ombilic du rêve, l'endroit où il repose sur l'inconnu. Les pensées du rêve auxquelles on a accès par l'interprétation doivent demeurer d'une manière générale sans clôture, et partir de tous côtés dans le réseau embrouillé du monde de nos pensées. D'un endroit plus dense de ce réseau surgit le désir du rêve comme le champignon de son mycélium». Freud ne reviendra pas sur cette façon de dire que l'interprétation est infinie, puisqu'en dernière analyse, il reste cet irréductible ombilic du rêve, qui demeure dans l'ombre et qui met en échec une interprétation exhaustive du rêve.

2. Pour construire cette hypothèse, je me suis inspiré des travaux de M. Bakhtine (1934-1935, 1963). Bakhtine a introduit la notion de polyphonie dans son analyse de la structure de l'œuvre littéraire. Il soutient l'idée que le roman polyphonique s'élabore au croisement d'autres structures, tout comme le mot est une polyphonie de plusieurs écritures: celle de l'écrivain, de ses personnages, du destinataire, du contexte historique, éthique et culturel. Cette organisation polyphonique caractérise la sphère même du langage et Bakhtine en étend le principe à toute production sémiotique : la logique qui l'organise n'est pas celle de la détermination linéaire et de l'identité, mais celle, transgressive, de la logique du Rêve ou de la Révolution: une autre loi y opère.

La référence à la notion de polyphonie dans la théorie musicale nous indique un agencement de plusieurs de voix ou de plusieurs sons. Toutefois, dans le contexte où j'utilise la notion de polyphonie, celle-ci comprend aussi la matière iconique et les formes visuelles du rêve. Il faut alors parler de polyiconie du rêve, ou encore de polyptyque, si l'on veut rendre compte des nombreux replis du rêve dans les tableaux (ou les scènes) qui se lient les uns aux autres. Par convention, nous admettrons que le terme de polyphonie, plus euphonique, assume toutes ces dimensions.

3. Cf. D. Anzieu, 1985.

\section{Références}

Anzieu D., 1985, Le Moi-peau, Paris, Dunod.

Bakhtine M., 1934-35, Le discours du roman, in Bakhtine M., Esthétique et théorie du roman, trad. fr., Paris, Gallimard (1978).

Bakhtine M., 1963, La poétique de Dostö̈evski, trad. fr., Paris, Les Éditions du Seuil (1970).

Freud S., 1900, Die Traumdeutung, G.-W., II-III, Frankfurt-am-Main, S. Fischer Verlag, 1-642.

Freud S., 1901, Über den Traum, G.-W., II-III, 643-700.

J. Irving, 1993, Les rêves des autres, trad. fr. Paris, Editions du Seuil.

Kaës R., 1993, Le sujet de l'héritage, in R. Kaës, H. Faimberg, M. Enriquez, J.-J. Baranes, Transmission de la vie psychique entre générations. Paris, Dunod.

Kaës R., 2002, La polyphonie du rêve. L'espace onirique commun et partagé, Paris, Dunod.

Saramago J., 1995, L'aveuglement, trad. fr. Paris, Les Éditions du Seuil (1997). 